\title{
New Fixed Point Theorems for Mixed Monotone Operators with Perturbation and Applications
}

\author{
Fengxia Zheng \\ Department of Mathematics, Sichuan University of Arts and Science, Dazhou, P. R. China
}

Email address:

zhengfengxiade@163.com

To cite this article:

Fengxia Zheng. New Fixed Point Theorems for Mixed Monotone Operators with Perturbation and Applications. International Journal of Theoretical and Applied Mathematics. Vol. 3, No. 6, 2017, pp. 182-190. doi: 10.11648/j.jitam.20170306.12

Received: September 29, 2017; Accepted: October 23, 2017; Published: November 15, 2017

\begin{abstract}
By using the properties of cone and the fixed point theorem for mixed monotone operators in ordered Banach spaces, we investigate the mixed monotone operators of a new type with perturbation. We establish some sufficient conditions for such operators to have a new existence and uniqueness fixed point and provide monotone iterative techniques which give sequences convergent to the fixed point. Finally, as applications, we apple the results obtained in this paper to study the existence and uniqueness of positive solutions for nonlinear fractional differential equation boundary value problems.
\end{abstract}

Keywords: Fixed Point, Mixed Monotone Operator, Positive Solution, Fractional Differential Equation, Boundary Value Problem

\section{Introduction and Preliminaries}

The study of mixed monotone operators has been a lot of discussion since they were introduced by Guo and Lakshmikantham (see [1]) in 1987, because they have not only important theoretical meaning but also wide applications in microeconomics, the nuclear industry, and so on (see $[1,2]$ ). In the past several decades, many authors investigated these kinds of operators in ordered Banach spaces and obtained a lot of interesting and important fixed point theorems for mixed monotone operators, see [3-5] and the references therein. Recently, some new results about these kinds of operators have emerged, and they are extensively used in nonlinear differential and integral equations, see [6-9, 26, 27] and the references therein. In this paper, without demanding the assumptions of the existence of coupled upper-lower solutions or compactness or continuity, we study mixed monotone operators with perturbation and give several of new fixed point theorems. In other words, we consider the existence and uniqueness of positive solutions to the following operator equation in ordered Banach spaces:

$$
A(x, x)+B x=x
$$

where $A$ is a mixed monotone operator, $B$ is an increasing sub-homogeneous operator or general $\alpha$-concave operator.
The results in essence extend and generalize recent related results, see [10-12] and the references therein. As an application, we apply our main fixed point theorem to study a class of nonlinear fractional differential equation boundary value problems.

Suppose $(E,\|\cdot\|)$ is a real Banach space which is partially ordered by a cone $P \subset E$, i.e. $x \leq y$ if and only if $y-x \in P$. If $x \leq y$ and $x \neq y$, then we denote $x<y$. We denote the zero element of $E$ by $\theta$. Recall that a non-empty closed convex set $P \subset E$ is a cone if it satisfies

(i) $x \in P, \lambda \geq 0 \Rightarrow \lambda x \in P$; (ii) $x \in P,-x \in P \Rightarrow x=\theta$.

Putting $P^{0}=\{x \in P \mid x$ is an interior point of $P\}$, a cone $P$ is said to be solid if $P^{0}$ is non-empty. Moreover, $P$ is called normal if there exists a constant $N>0$ such that, for all $x, y \in E, \theta \leq x \leq y$ implies $\|x\| \leq N\|y\|$; in this case $N$ is called the normality constant of $P$.

We say that an operator $A: E \rightarrow E$ is increasing if $x \leq y$ implies $A x \leq A y$. Element $x \in P$ is called a fixed point of $A$ if $A x=x$.

For all $x, y \in E$, the notation $x \sim y$ means that there exist $\lambda>0$ and $\mu>0$ such that $\lambda x \leq y \leq \mu x$. Clearly $\sim$ is an equivalence relation. Given $w>\theta$ (i.e. $w \geq \theta$ and $w \neq \theta$ ), we denote the set $P_{w}=\{x \in E \mid x \sim w\}$ by $P_{w}$. It is easy to see that $P_{w} \subset P$ for $w \in P$. 
All the concepts discussed above can be found in [2, 12-15]. For more results about mixed monotone operators and other related concepts, the reader is referred to [10-12] and some of the references therein.

Definition 1.1 (see [1]) An operator $A: P \rightarrow P$ is said to be a mixed monotone operator if $A(x, y)$ is increasing in $x$ and decreasing in $y$. Element $x \in P$ is called a fixed point of $A$ if $A(x, x)=x$.

Definition 1.2 (see [12]) An operator $A: P \rightarrow P$ is said to be a sub-homogeneous operator if it satisfies:

$$
A(t x) \geq t A x, \quad \forall t \in(0,1), x \in P .
$$

Definition 1.3 (see [16]) An operator $A: P \rightarrow P$ is said to be a general $\alpha$-concave operator if it satisfies: for all $x \in P$ and $t \in(0,1)$, there exists $0<\alpha(t)<1$ such that $A(t x) \geq t^{\alpha(t)} A x$.

Definition 1.4 (see [17]) An operator $A: P \times P \rightarrow P$ is said to be a $t-\alpha(t)$ mixed monotone model operator if it satisfies: for all $x, y \in P$ and $t \in(0,1)$, there exists $0<\alpha(t)<1$ such that $A\left(t x, \frac{1}{t} y\right) \geq t^{\alpha(t)} A(x, y)$.

Lemma 1.5 (see [9]) Let $P$ be a normal cone in $E$. Assume that $T: P \times P \rightarrow P$ is a mixed monotone operator and satisfies:

(A1) there exists $w \in P$ with $w \neq \theta$ such that $T(w, w) \in P_{w}$;

(A2) for any $u, v \in P$ and $t \in(0,1)$, there exists $\phi(t) \in(t, 1]$ such that $T\left(t u, \frac{1}{t} v\right) \geq \phi(t) T(u, v)$.

Then

(T1) $T: P_{w} \times P_{w} \rightarrow P_{w}$;

(T2) there exist $u_{0}, v_{0} \in P_{w}$ and $r \in(0,1)$ such that

$$
r v_{0} \leq u_{0}<v_{0}, u_{0} \leq T\left(u_{0}, v_{0}\right) \leq T\left(v_{0}, u_{0}\right) \leq v_{0} ;
$$

(T3) $T$ has a unique fixed point $x *$ in $P_{w}$;

(T4) for any initial values $x_{0}, y_{0} \in P_{w}$, constructing successively the sequences

$$
x_{n}=T\left(x_{n-1}, y_{n-1}\right), \quad y_{n}=T\left(y_{n-1}, x_{n-1}\right), \quad n=1,2, \ldots,
$$

we have $x_{n} \rightarrow x^{*}$ and $y_{n} \rightarrow x^{*}$ as $n \rightarrow \infty$.

\section{Main Results}

In this section, we present our main results. We always assume that $E$ is a real Banach space with a partial order introduced by a normal cone $P$ of $E$. Take $w \in E, w>\theta, P_{w}$ is given as in the first part.

Theorem 2.1 $A: P \times P \rightarrow P$ is a mixed monotone operator and satisfies

$$
A\left(t x, \frac{1}{t} y\right) \geq t^{\alpha(t)} A(x, y), \quad t \in(0,1), x, y \in P,
$$

where the function $\alpha(t)$ is differentiated in the interval $(0,1)$ and $0<\alpha(t)<1$.

$B: P \rightarrow P$ is an increasing sub-homogeneous operator.

Assume that

(i) there is $w_{0} \in P_{w}$ such that $A\left(w_{0}, w_{0}\right) \in P_{w}$ and $B w_{0} \in P_{w}$;

(ii) there exists a constant $\delta_{0}>0$ such that $A(x, y) \geq \delta_{0} B x, \forall x, y \in P$.

Then

(T1) $A: P_{w} \times P_{w} \rightarrow P_{w}, B: P_{w} \rightarrow P_{w}$;

(T2) there exist $u_{0}, v_{0} \in P_{w}$ and $r \in(0,1)$ such that

$r v_{0} \leq u_{0}<v_{0}, \quad u_{0} \leq A\left(u_{0}, v_{0}\right)+B u_{0} \leq A\left(v_{0}, u_{0}\right)+B v_{0} \leq v_{0}$;

(T3) the operator equation (1) has a unique solution $x^{*}$ in $P_{w}$;

(T4) for any initial values $x_{0}, y_{0} \in P_{w}$, constructing successively the sequences we have $x_{n} \rightarrow x^{*}$ and $y_{n} \rightarrow x^{*}$ as $n \rightarrow \infty$.

Proof: Firstly, for $t \in(0,1), x, y \in P$, from (2) and (3), we have

$$
\begin{gathered}
A(x, y)=A\left(t \cdot \frac{1}{t} x, \frac{1}{t} \cdot t y\right) \geq t^{\alpha(t)} A\left(\frac{1}{t} x, t y\right), x_{n}=A\left(x_{n-1}, y_{n-1}\right)+B x_{n-1}, \quad y_{n}=A\left(y_{n-1}, x_{n-1}\right)+B y_{n-1}, \quad n=1,2, \ldots \\
B x=B\left(t \cdot \frac{1}{t} x\right) \geq t B\left(\frac{1}{t} x\right)
\end{gathered}
$$

Hence

$$
A\left(\frac{1}{t} x, t y\right) \leq \frac{1}{t^{\alpha(t)}} A(x, y) \text { and } B\left(\frac{1}{t} x\right) \leq \frac{1}{t} B x \text { for } t \in(0,1), x, y \in P
$$

Since there is $w_{0} \in P_{w}$ such that $A\left(w_{0}, w_{0}\right) \in P_{w}$ and $B w_{0} \in P_{w}$, there exist constants $\lambda_{1}, \lambda_{2}, v_{1}, v_{2}>0$ such that

$$
\lambda_{1} w \leq A\left(w_{0}, w_{0}\right) \leq \lambda_{2} w, \quad v_{1} w \leq B w_{0} \leq v_{2} w .
$$

Also from $w_{0} \in P_{w}$, there exists a constant $t_{0} \in(0,1)$ such

Then from (3) and (4) and the mixed monotone properties of operator $A$, we have that 


$$
\begin{aligned}
& A(w, w) \leq A\left(\frac{1}{t_{0}} w_{0}, t_{0} w_{0}\right) \leq \frac{1}{t_{0}}{ }^{\alpha\left(t_{0}\right)} A\left(w_{0}, w_{0}\right) \leq \frac{\lambda_{2}}{t_{0}{ }^{\alpha\left(t_{0}\right)}} w \\
& A(w, w) \geq A\left(t_{0} w_{0}, \frac{1}{t_{0}} w_{0}\right) \geq t_{0}{ }^{\alpha\left(t_{0}\right)} A\left(w_{0}, w_{0}\right) \geq \lambda_{1} t_{0}{ }^{\alpha\left(t_{0}\right)} w .
\end{aligned}
$$

Noting that $\frac{\lambda_{2}}{t_{0}^{\alpha\left(t_{0}\right)}}, \lambda_{1} t_{0}{ }^{\alpha\left(t_{0}\right)}>0$, we can get $A(w, w) \in P_{w}$. An application of Lemma 1.5 implies that $A: P_{w} \times P_{w} \rightarrow P_{w}$. And from (2), (4) and the monotone property of operator $B$, we have

$$
B w \leq B\left(\frac{1}{t_{0}} w_{0}\right) \leq \frac{1}{t_{0}} B w_{0} \leq \frac{v_{2}}{t_{0}} w, \quad B w \geq B\left(t_{0} w_{0}\right) \geq t_{0} B w_{0} \geq v_{1} t_{0} w .
$$

Next we show $B: P_{w} \rightarrow P_{w}$. For any $x \in P_{w}$; we can choose a sufficiently small number $\mu \in(0,1)$ such that

$$
\mu w \leq x \leq \frac{1}{\mu} w .
$$

Since $B\left(\frac{1}{\mu} w\right) \leq \frac{1}{\mu} B(w)$ and $B w \leq B\left(\frac{1}{t_{0}} w_{0}\right) \leq \frac{1}{t_{0}} B w_{0} \leq \frac{v_{2}}{t_{0}} w$, then

$$
B x \leq B\left(\frac{1}{\mu} w\right) \leq \frac{1}{\mu} \cdot \frac{v_{2}}{t_{0}} w, B x \geq B(\mu w) \geq \mu t_{0} v_{1} w .
$$

Evidently, $\frac{v_{2}}{\mu t_{0}} w, \mu t_{0} v_{1}>0$. Thus $B x \in P_{w} ;$ that is, $B: P_{w} \rightarrow P_{w}$. So the conclusion (1) holds.

Now we define an operator $T=A+B$ by $T(x, y)=A(x, y)+B x$. Then $T: P \times P \rightarrow P$ is a mixed monotone operator and $T(w, w) \in P_{w}$. In the following, we show that there exists $\phi(t) \in(t, 1]$ with respect to $t \in(0,1)$ such that $T\left(t x, \frac{1}{t} y\right) \geq \phi(t) T(x, y), \quad \forall x, y \in P$.

Let $\bar{\alpha}=\sup _{0<t<1} \alpha(t), \alpha_{1}=\lim _{\mathrm{t} \rightarrow 1^{-}} \alpha(t)$. Consider the following function:

$$
f(t)=\frac{t^{\beta}-t}{t^{\alpha(t)}-t^{\beta}}, \quad \forall t \in(0,1), \text { where } \beta \in(\bar{\alpha}, 1) .
$$

It is easy to prove that $f$ is increasing in $(0,1)$ and

$$
\lim _{t \rightarrow 0^{+}} f(t)=0, \quad \lim _{t \rightarrow 1^{-}} f(t)=\frac{\beta-1}{\alpha_{1}-\beta}>0 .
$$

Further, fixing $t \in(0,1)$, we have

$$
\lim _{\beta \rightarrow 1^{-}} f(t)=\lim _{\beta \rightarrow 1^{-}} \frac{t^{\beta}-t}{t^{\alpha(t)}-t^{\beta}}=0 .
$$

So there exists $\beta_{0}(t) \in(\bar{\alpha}, 1)$ with respect to $t$ such that

$$
\frac{t^{\beta_{0}(t)}-t}{t^{\alpha(t)}-t^{\beta_{0}(t)}} \leq \delta_{0}, \quad t \in(0,1) \text {. }
$$

Hence we have

$$
A(x, y) \geq \delta_{0} B x \geq \frac{t^{\beta_{0}(t)}-t}{t^{\alpha(t)}-t^{\beta_{0}(t)}} B x, \quad \forall t \in(0,1), x, y \in P .
$$

Then we obtain

$\left.t^{\alpha(t)} A(x, y)+t B x \geq t^{\beta_{0}(t)}[A(x, y)+B x], \quad \forall t \in(0,1), x, y \in P\right]$.

Consequently, for any $t \in(0,1)$ and $x, y \in P$,

$$
T\left(t x, \frac{1}{t} y\right)=A\left(t x, \frac{1}{t} y\right)+B(t x) \geq t^{\alpha(t)} A(x, y)+t B(x) \geq t^{\beta_{0}(t)}[A(x, y)+B x]=t^{\beta_{0}(t)} T(x, y) .
$$

Let $\phi(t)=t^{\beta_{0}(t)}, t \in(0,1) \quad$. Then $\quad \phi(t) \in(t, 1] \quad$ and $T\left(t x, \frac{1}{t} y\right) \geq \phi(t) T(x, y)$ for any $t \in(0,1)$ and $x, y \in P$. Hence the condition (A2) in Lemma 1.5 is satisfied. An application of Lemma 1.5 implies:

(c1) there exist $u_{0}, v_{0} \in P_{w}$ and $r \in(0,1)$ such that $r v_{0} \leq u_{0}<v_{0}, u_{0} \leq T\left(u_{0}, v_{0}\right) \leq T\left(v_{0}, u_{0}\right) \leq v_{0} ;$

(c2) $T$ has a unique fixed point $x *$ in $P_{w}$;

(c3) for any initial values $x_{0}, y_{0} \in P_{w}$, constructing successively the sequences

$$
x_{n}=T\left(x_{n-1}, y_{n-1}\right), \quad y_{n}=T\left(y_{n-1}, x_{n-1}\right), \quad n=1,2, \ldots,
$$

we have $x_{n} \rightarrow x^{*}$ and $y_{n} \rightarrow x^{*}$ as $n \rightarrow \infty$. That is, the conclusions (T2) -(T4) hold.

From the proof of Theorem 2.1 , we can easily prove the following corollary.
Corollary 2.2 Let the function $\alpha(t)$ be differentiated in the interval $(0,1)$ and $0<\alpha(t)<1$.

$A: P \times P \rightarrow P$ is a mixed monotone operator. Assume that

(3) holds and there is $w_{0}>\theta$ such that $A\left(w_{0}, w_{0}\right) \in P_{w}$.

Then

(T1) $A: P_{w} \times P_{w} \rightarrow P_{w}$;

(T2) there exist $u_{0}, v_{0} \in P_{w}$ and $r \in(0,1)$ such that $r v_{0} \leq u_{0}<v_{0}, \quad u_{0} \leq A\left(u_{0}, v_{0}\right) \leq A\left(v_{0}, u_{0}\right) \leq v_{0}$;

(T3) the operator equation $A(x, x)=x$ has a unique solution $x *$ in $P_{w}$;

(T4) for any initial values $x_{0}, y_{0} \in P_{w}$, constructing successively the sequences

$$
x_{n}=A\left(x_{n-1}, y_{n-1}\right), \quad y_{n}=A\left(y_{n-1}, x_{n-1}\right), \quad n=1,2, \ldots,
$$

we have $x_{n} \rightarrow x^{*}$ and $y_{n} \rightarrow x^{*}$ as $n \rightarrow \infty$.

Corollary 2.3 Let the function $\alpha(t)$ be differentiated in the 
interval $(0,1)$ and $0<\alpha(t)<1$.

$A: P \times P \rightarrow P$ is a mixed monotone operator and $B: P \rightarrow P$ is an increasing sub-homogeneous operator. Assume that (3) holds and

(i) there is $w_{0}>\theta$ such that $A\left(w_{0}, w_{0}\right) \in P_{w}$ and $B w_{0} \in P_{w}$;

(ii) there exists a constant $\delta_{0}>0$ such that

$$
x_{n}=\frac{1}{\lambda}\left[A\left(x_{n-1}, y_{n-1}\right)+B x_{n-1}\right], \quad y_{n}=\frac{1}{\lambda}\left[A\left(y_{n-1}, x_{n-1}\right)+B y_{n-1}\right], \quad n=1,2, \ldots,
$$

we have $x_{n} \rightarrow x_{\lambda}^{*}$ and $y_{n} \rightarrow x_{\lambda}^{*}$ as $n \rightarrow \infty$.

Theorem $2.4 A: P \times P \rightarrow P$ is a mixed monotone operator and satisfies

$$
A\left(t x, \frac{1}{t} y\right) \geq t A(x, y), \quad t \in(0,1), x, y \in P .
$$

$B: P \rightarrow P$ is an increasing general $\alpha$-concave operator and satisfies

$$
B(t x) \geq t^{\alpha(t)} B x, \quad t \in(0,1), x, y \in P,
$$

where the function $\alpha(t)$ be differentiated in the interval $(0,1)$ and $0<\alpha(t)<1$.

Assume that

(i) there is $w_{0} \in P_{w}$ such that $A\left(w_{0}, w_{0}\right) \in P_{w}$ and $B w_{0} \in P_{w}$;

(ii) there exists a constant $\delta_{0}>0$ such that $A(x, y) \leq \delta_{0} B x, \forall x, y \in P$.

Then

(T1) $A: P_{w} \times P_{w} \rightarrow P_{w}, B: P_{w} \rightarrow P_{w}$;

(T2) there exist $u_{0}, v_{0} \in P_{w}$ and $r \in(0,1)$ such that $r v_{0} \leq u_{0}<v_{0}, \quad u_{0} \leq A\left(u_{0}, v_{0}\right)+B u_{0} \leq A\left(v_{0}, u_{0}\right)+B v_{0} \leq v_{0} ;$

(T3) the operator equation (1) has a unique solution $x *$ in $P_{w}$

(T4) for any initial values $x_{0}, y_{0} \in P_{w}$, constructing successively the sequences

$$
x_{n}=A\left(x_{n-1}, y_{n-1}\right)+B x_{n-1}, \quad y_{n}=A\left(y_{n-1}, x_{n-1}\right)+B y_{n-1}, \quad n=1,2, \ldots,
$$

we have $x_{n} \rightarrow x *$ and $y_{n} \rightarrow x *$ as $n \rightarrow \infty$.

Proof: Firstly, from (5) and (6), we have

$$
A\left(\frac{1}{t} x, t y\right) \leq \frac{1}{t} A(x, y) \text { and } B\left(\frac{1}{t} x\right) \leq \frac{1}{t^{\alpha(t)}} B x \text { for } t \in(0,1), x, y \in P .
$$

Next, we define an operator $T=A+B$ by $T(x, y)=A(x, y)+B x$. Similarly to the proof of Theorem 2.1, we have $A: P_{w} \times P_{w} \rightarrow P_{w}, B: P_{w} \rightarrow P_{w}$. Further, we can easily prove that $T: P \times P \rightarrow P$ is a mixed monotone operator and $T(w, w) \in P_{w}$.

In the following, we show that there exists $\varphi(t) \in(t, 1]$ with respect to $t \in(0,1)$ such that

$$
T\left(t x, \frac{1}{t} y\right) \geq \varphi(t) T(x, y), \quad \forall x, y \in P
$$

Let $\bar{\alpha}=\sup _{0<t<1} \alpha(t), \alpha_{1}=\lim _{\mathrm{t} \rightarrow \mathrm{I}^{-}} \alpha(t)$. Consider the following function:

$$
f(t)=\frac{t^{\alpha(t)}-t^{\beta}}{t^{\beta}-t}, \quad \forall t \in(0,1) \text {, where } \beta \in(\bar{\alpha}, 1) .
$$

It is easy to prove that $f$ is decreasing in $(0,1)$ and

$$
\lim _{t \rightarrow 0^{+}} f(t)=+\infty, \quad \lim _{t \rightarrow 1^{-}} f(t)=\frac{\alpha_{1}-\beta}{\beta-1}>0 .
$$

Further, fixing $t \in(0,1)$, we have

$$
\lim _{\beta \rightarrow 1^{-}} f(t)=\lim _{\beta \rightarrow 1^{-}} \frac{t^{\alpha(t)}-t^{\beta}}{t^{\beta}-t}=+\infty .
$$

So there exists $\beta_{0}(t) \in(\bar{\alpha}, 1)$ with respect to $t$ such that

$$
\frac{t^{\alpha(t)}-t^{\beta_{0}(t)}}{t^{\beta_{0}(t)}-t} \geq \delta_{0}, \quad t \in(0,1) .
$$

Hence we have

$$
A(x, y) \leq \delta_{0} B x \leq \frac{t^{\alpha(t)}-t^{\beta_{0}(t)}}{t^{\beta_{0}(t)}-t} B x, \quad \forall t \in(0,1), x, y \in P
$$

Then we obtain

$t A(x, y)+t^{\alpha(t)} B x \geq t^{\beta_{0}(t)}[A(x, y)+B x], \quad \forall t \in(0,1), x, y \in P$.

Consequently, for any $t \in(0,1)$ and $x, y \in P$, 


$$
T\left(t x, \frac{1}{t} y\right)=A\left(t x, \frac{1}{t} y\right)+B(t x) \geq t A(x, y)+t^{\alpha(t)} B(x) \geq t^{\beta_{0}(t)}[A(x, y)+B x]=t^{\beta_{0}(t)} T(x, y) .
$$

Let $\varphi(t)=t^{\beta_{0}(t)}, t \in(0,1) \quad$. Then $\quad \varphi(t) \in(t, 1] \quad$ and $T\left(t x, \frac{1}{t} y\right) \geq \varphi(t) T(x, y)$ for any $t \in(0,1)$ and $x, y \in P$. Hence the condition (A2) in Lemma 1.5 is satisfied. An application of Lemma 1.5 implies:

$\left(\mathrm{c}_{1}\right)$ there exist $u_{0}, v_{0} \in P_{w}$ and $r \in(0,1)$ such that $r v_{0} \leq u_{0}<v_{0}, u_{0} \leq T\left(u_{0}, v_{0}\right) \leq T\left(v_{0}, u_{0}\right) \leq v_{0} ;$

( $\left.\mathrm{c}_{2}\right) T$ has a unique fixed point $x *$ in $P_{w}$;

$\left(c_{3}\right)$ for any initial values $x_{0}, y_{0} \in P_{w}$, constructing successively the sequences

$$
x_{n}=T\left(x_{n-1}, y_{n-1}\right), \quad y_{n}=T\left(y_{n-1}, x_{n-1}\right), \quad n=1,2, \ldots,
$$

we have $x_{n} \rightarrow x^{*}$ and $y_{n} \rightarrow x^{*}$ as $n \rightarrow \infty$. That is, the conclusions (T2) -(T4) hold.

From the proof of Theorem 2.4 , we can easily prove the following conclusion.

Corollary 2.5 $A: P \times P \rightarrow P$ is a mixed monotone operator and $B: P \rightarrow P$ is an increasing general $\alpha$-concave operator.

Assume that (5), (6) hold and

(i) there is $w_{0}>\theta$ such that $A\left(w_{0}, w_{0}\right) \in P_{w}$ and $B w_{0} \in P_{w}$;

(ii) there exists a constant $\delta_{0}>0$ such that $A(x, y) \leq \delta_{0} B x, \forall x, y \in P$.

Then the operator equation $A(x, x)+B x=\lambda x$ has a unique solution $x_{\lambda}^{*}$ in $P_{w}$ for any given $\lambda>0$.

Moreover, for any initial values $x_{0}, y_{0} \in P_{w}$, constructing successively the sequences

$$
x_{n}=\frac{1}{\lambda}\left[A\left(x_{n-1}, y_{n-1}\right)+B x_{n-1}\right], \quad y_{n}=\frac{1}{\lambda}\left[A\left(y_{n-1}, x_{n-1}\right)+B y_{n-1}\right], \quad n=1,2, \ldots,
$$

we have $x_{n} \rightarrow x_{\lambda}^{*}$ and $y_{n} \rightarrow x_{\lambda}^{*}$ as $n \rightarrow \infty$.

Remark 2.6 Take $w \in P^{0}$. If we suppose that operator $A: P_{w} \times P_{w} \rightarrow P_{w}$ or

$A: P^{0} \times P^{0} \rightarrow P^{0}$ with $P$ is a solid cone, then $A\left(w_{0}, w_{0}\right) \in P_{w}$ for any $w_{0} \in P_{w}$ (or $P^{0}$ ) is automatically satisfied.

Let $D=P_{w}$ or $P^{0}$. Then we have

Corollary 2.7 $A: D \times D \rightarrow D$ is a mixed monotone operator and $B: D \rightarrow D$ is an increasing sub-homogeneous operator. Assume that (3) holds and there exists a constant

$$
x_{n}=A\left(x_{n-1}, y_{n-1}\right)+B x_{n-1}, \quad y_{n}=A\left(y_{n-1}, x_{n-1}\right)+B y_{n-1}, \quad n=1,2, \ldots,
$$

we have $x_{n} \rightarrow x^{*}$ and $y_{n} \rightarrow x *$ as $n \rightarrow \infty$.

Corollary $2.8 A: D \times D \rightarrow D$ is a mixed monotone operator and $B: D \rightarrow D$ is an increasing general $\alpha$-concave operator. Assume that (5), (6) hold and there exists a constant $\delta_{0}>0$ such that $A(x, y) \leq \delta_{0} B x, \forall x, y \in D$. Then

(T1) there exist $u_{0}, v_{0} \in P_{w}$ and $r \in(0,1)$ such that

$$
x_{n}=A\left(x_{n-1}, y_{n-1}\right)+B x_{n-1}, \quad y_{n}=A\left(y_{n-1}, x_{n-1}\right)+B y_{n-1}, \quad n=1,2, \ldots,
$$

we have $x_{n} \rightarrow x^{*}$ and $y_{n} \rightarrow x^{*}$ as $n \rightarrow \infty$.

\section{Applications}

Fractional differential equations have been of great interest recently. It is caused both by the intensive development of the theory of fractional calculus itself and by the applications of such constructions in various sciences such as physics, mechanics, chemistry, engineering, etc. For details, see $[17,18]$ and references therein. In this section, we use the results in section 2 to study the existence of a unique positive solution for nonlinear fractional differential equation boundary value problem:

$$
\left\{\begin{array}{l}
-D_{0+}^{\alpha} u(t)=f(t, u(t), u(t))+g(t, u(t)), 0<t<1 \\
u(0)=u(1)=0
\end{array}\right.
$$

where $1<\alpha \leq 2$ is a real number and $D_{a+}^{\alpha}$ is the RiemannLiouville fractional derivative of order $\alpha>0$, defined by 


$$
D_{0+}^{\alpha} u(t)=\frac{1}{\Gamma(n-\alpha)}\left(\frac{\mathrm{d}}{\mathrm{d} t}\right)^{n} \int_{0}^{t} \frac{u(\tau)}{(t-\tau)^{\alpha-n+1}} \mathrm{~d} \tau,
$$

where $n=[\alpha]+1,[\alpha]$ denotes the integer part of number $\alpha$, see $[19,20]$.

In recent years, there are many papers discuss the existence and multiplicity of positive solutions for nonlinear fractional differential equation boundary value problem by the use of Leray-Shauder theory, fixed-point theorems, etc., see [21-25]. However, there are few papers consider the existence of a unique positive solution for nonlinear fractional differential equation boundary value problem. In this section, we apply the results in section 2 to study the nonlinear fractional differential equation boundary value problem (8).

Let $E=C[0,1]$ be a Banach space of continuous functions on $[0,1]$ with the maximum norm

$\| u \mid=\max \{|u(t)|: t \in[0,1]\}$

$P=\{u \in E \mid u(t) \geq 0, \forall t \in[0,1]\}$, then $P$ is a normal solid cone of which the normality constant is 1 in Banach space $E$. The partial ordering defined by $P$ is given by $u \leq v \Leftrightarrow u(t) \leq v(t)$ for all $t \in[0,1]$.
Lemma 3.1 (see [21]) Given $y \in C[0,1]$ and $1<\alpha \leq 2$, the unique solution of

$$
\left\{\begin{array}{l}
-D_{0+}^{\alpha} u(t)=y(t), 0<t<1 \\
u(0)=u(1)=0
\end{array}\right.
$$

is

$$
u(t)=\int_{0}^{1} G(t, s) y(s) \mathrm{d} s
$$

where

$$
G(t, s)= \begin{cases}\frac{[t(1-s)]^{\alpha-1}-(t-s)^{\alpha-1}}{\Gamma(\alpha)}, & 0 \leq s \leq t \leq 1, \\ \frac{[t(1-s)]^{\alpha-1}}{\Gamma(\alpha)}, & 0 \leq t \leq s \leq 1 .\end{cases}
$$

Moreover, $G(t, s) \geq 0$ for $\forall t, s \in(0,1)$.

Lemma 3.2 (see [22]) The Green function $G(t, s)$ in Lemma 3.1 has the following property:

$$
\frac{\alpha-1}{\Gamma(\alpha)} t^{\alpha-1}(1-t)(1-s)^{\alpha-1} s \leq G(t, s) \leq \frac{1}{\Gamma(\alpha)} t^{\alpha-1}(1-t)(1-s)^{\alpha-2} \text { for } t, s \in(0,1)
$$

Theorem 3.3 Assume that

(H1) $\quad f(t, u, v):[0,1] \times[0,+\infty) \times[0,+\infty) \rightarrow[0,+\infty) \quad$ is continuous and $g(t, u):[0,1] \times[0,+\infty) \rightarrow[0,+\infty)$ is continuous with $g(t, 0)$ 丰 0 ;

(H2) $f(t, u, v)$ is increasing in $u \in[0,+\infty)$ for fixed $t \in[0,1]$ and $v \in[0,+\infty)$, decreasing in $v \in[0,+\infty)$ for fixed $t \in[0,1]$ and $u \in[0,+\infty)$, and $g(t, u)$ is increasing in $u \in[0,+\infty)$ for fixed $t \in[0,1]$;

(H3) there exists a function $\alpha(t)$ which is differentiated in the interval $(0,1)$ and $0<\alpha(t)<1$

such that

$$
f\left(t, \lambda u, \frac{1}{\lambda} v\right) \geq \lambda^{\alpha(\lambda)} f(t, u, v), \forall t \in[0,1], \lambda \in(0,1), u, v \in[0,+\infty)
$$

and

$$
g(t, \mu u) \geq \mu g(t, u), \quad \mu \in(0,1), t \in[0,1], u \in[0,+\infty) ;
$$

(H4) there exists a constant $\delta_{0}>0$ such that $f(t, u, v) \geq \delta_{0} g(t, u), t \in[0,1], u, v \geq 0$.

Then the problem (8) has a unique positive solution $u *$ in $P_{w}$, where $w(t)=t^{\alpha-1}(1-t), t \in[0,1]$. Moreover, for any initial value $x_{0}, y_{0} \in P_{w}$, constructing successively the iterative scheme

$$
\begin{aligned}
& x_{n}(t)=\int_{0}^{1} G(t, s) f\left(s, x_{n-1}(s), y_{n-1}(s)\right) \mathrm{d} s+\int_{0}^{1} G(t, s) g\left(s, x_{n-1}(s)\right) \mathrm{d} s, \quad n=1,2, \cdots, \\
& y_{n}(t)=\int_{0}^{1} G(t, s) f\left(s, y_{n-1}(s), x_{n-1}(s)\right) \mathrm{d} s+\int_{0}^{1} G(t, s) g\left(s, y_{n-1}(s)\right) \mathrm{d} s, \quad n=1,2, \cdots,
\end{aligned}
$$

we have $x_{n} \rightarrow u^{*}$ and $y_{n} \rightarrow u^{*}$ as $n \rightarrow \infty$, where $G(t, s)$ is given as (10).

Proof. To begin with, from Lemma 3.1, the problem (8) has an integral formulation given by

$$
u(t)=\int_{0}^{1} G(t, s)[f(s, u(s), u(s))+g(s, u(s))] \mathrm{d},
$$

where $G(t, s)$ is given as in Lemma 3.1.

Define two operators $A: P \times P \rightarrow E$ and $B: P \rightarrow E$ by

$$
A(u, v)(t)=\int_{0}^{1} G(t, s) f(s, u(s), v(s)) \mathrm{d} s, \quad B u(t)=\int_{0}^{1} G(t, s) g(s, u(s)) \mathrm{d} s .
$$

It is easy to prove that $u$ is the solution of the problem (8) if and only if $u=A(u, u)+B u$.

By assumption (H1) and Lemma 3.2, we know that $A: P \times P \rightarrow P$ and $B: P \rightarrow P$. Further, it follows from (H2) that $A$ is 
mixed monotone and $B$ is increasing. For any $\lambda \in(0,1)$ and $u, v \in P$, from (H3) we know that

$$
A\left(\lambda u, \frac{1}{\lambda} v\right)(t)=\int_{0}^{1} G(t, s) f\left(s, \lambda u(s), \frac{1}{\lambda} v(s)\right) \mathrm{d} s \geq \lambda^{\alpha(\lambda)} \int_{0}^{1} G(t, s) f(s, u(s), v(s)) \mathrm{d} s=\lambda^{\alpha(\lambda)} A(u, v)(t) .
$$

That is, $A\left(\lambda u, \frac{1}{\lambda} v\right) \geq \lambda^{\alpha(\lambda)} A(u, v)$ for $\lambda \in(0,1), u, v \in P$. So the operator satisfies (3). Also, for any $\mu \in(0,1)$ and $u \in P$, by (H3) we obtain

$$
B(\mu u)(t)=\int_{0}^{1} G(t, s) g(s, \mu u(s)) \mathrm{d} s \geq \mu \int_{0}^{1} G(t, s) g(s, u(s)) \mathrm{d} s=\mu B u(t),
$$

That is, $B(\mu u) \geq \mu B u$ for $\mu \in(0,1), u \in P$. So the operator $B$ is a sub-homogeneous operator.

Next we show that $A(w, w) \in P_{w}$ and $B w \in P_{w}$, where $w(t)=t^{\alpha-1}(1-t)$.

By $(\mathrm{H} 1)$ and Lemma 3.2,

$$
\begin{aligned}
& A(w, w)(t)=\int_{0}^{1} G(t, s) f(s, w(s), w(s)) \mathrm{d} s \leq \frac{1}{\Gamma(\alpha)} w(t) \int_{0}^{1}(1-s)^{\alpha-2} f\left(s, w_{\text {max }}, 0\right) \mathrm{d} s \\
& A(w, w)(t)=\int_{0}^{1} G(t, s) f(s, w(s), w(s)) \mathrm{d} s \geq \frac{\alpha-1}{\Gamma(\alpha)} w(t) \int_{0}^{1} s(1-s)^{\alpha-1} f\left(s, 0, w_{\text {max }}\right) \mathrm{d} s,
\end{aligned}
$$

where $w_{\max }=\max \{w(t): t \in[0,1]\}$.

From $(\mathrm{H} 2)$ and $(\mathrm{H} 4)$, we have

$$
f\left(s, w_{\max }, 0\right) \geq f\left(s, 0, w_{\max }\right) \geq \delta_{0} g(s, 0) \geq 0 .
$$

Since $g(t, 0) \not \equiv 0$, we can get

$$
\int_{0}^{1} f\left(s, w_{\max }, 0\right) \mathrm{d} s \geq \int_{0}^{1} f\left(s, 0, w_{\max }\right) \mathrm{d} s \geq \delta_{0} \int_{0}^{1} g(s, 0) \mathrm{d} s>0
$$

and in consequence,

$$
l_{1}:=\frac{\alpha-1}{\Gamma(\alpha)} \int_{0}^{1} s(1-s)^{\alpha-1} f\left(s, 0, w_{\max }\right) \mathrm{d} s>0, l_{2}:=\frac{1}{\Gamma(\alpha)} \int_{0}^{1}(1-s)^{\alpha-2} f\left(s, w_{\max }, 0\right) \mathrm{d} s>0 .
$$

So $l_{1} w(t) \leq A w(t) \leq l_{2} w(t), t \in[0,1]$, and hence we have $A(w, w) \in P_{w}$. Similarly,

$$
\frac{\alpha-1}{\Gamma(\alpha)} w(t) \int_{0}^{1} s(1-s)^{\alpha-1} g(s, 0) \mathrm{d} s \leq B w(t) \leq \frac{1}{\Gamma(\alpha)} w(t) \int_{0}^{1}(1-s)^{\alpha-2} g\left(s, h_{\max }\right) \mathrm{d} s,
$$

from $g(t, 0) \not \equiv 0$, we easily prove $B w \in P_{w}$. Hence the condition (i) of Theorem 2.1 is satisfied.

In the following we show that the condition (ii) of Theorem 2.1 is satisfied.

For $u, v \in P$, by (H4),

$$
A(u, v)(t)=\int_{0}^{1} G(t, s) f(s, u(s), v(s)) \mathrm{d} s \geq \delta_{0} \int_{0}^{1} G(t, s) g(s, u(s)) \mathrm{d} s=\delta_{0} B u(t)
$$

Then we get $A(u, v) \geq \delta_{0} B u, u, v \in P$. So the conclusion of Theorem 3.3 follows from Theorem 2.1.

Remark 3.4 There exist many functions which satisfy the conditions of Theorem 3.3. For example $f(t, u, v)=t^{3}+u^{\alpha(\|u\|)}+v^{-\alpha(\|v\|)}, g(t, u)=t^{3}+u^{\alpha(\|u\|)}$, where $\alpha:(0,+\infty) \rightarrow(0,1)$ is non-decreasing.

Example 3.5 We give an example to illustrate Theorem 3.3. Consider the following nonlinear fractional differential equation boundary value problem:

$$
\left\{\begin{array}{l}
-D_{0+}^{\alpha} u(t)=2 t^{3}+2 u^{\beta(\|u\|)}+v^{-\beta(\|v\|)}, 0<t<1,1<\alpha \leq 2 \\
u(0)=u(1)=0
\end{array}\right.
$$

where $\beta:(0,+\infty) \rightarrow(0,1)$ is non-decreasing. In this example, we have 


$$
f(t, u, v)=t^{3}+u^{\beta(\|u\|)}+v^{-\beta(\|v\|)}, g(t, u)=t^{3}+u^{\beta(\|u\|)} .
$$

It is easy to show that the nonlinear fractional differential equation boundary value problem satisfy the conditions of Theorem 3.3. So the equation (12) has a unique positive solution $u^{*} \in P_{w}$, where $w(t)=t^{\alpha-1}(1-t)$.

From Theorem 3.3 and using Corollary 2.2, we can easily obtain the following result.

Theorem 3.4 Assume that

$$
f(t, u, v):[0,1] \times[0,+\infty) \times[0,+\infty) \rightarrow[0,+\infty)
$$
continuous with $f\left(t, 0, w_{\max }\right) \not \equiv 0$;

(P2) $f(t, u, v)$ is increasing in $u \in[0,+\infty)$ for fixed $t \in[0,1]$ and $v \in[0,+\infty)$, decreasing in $v \in[0,+\infty)$ for fixed $t \in[0,1]$ and $u \in[0,+\infty)$;

(P3) there exists a function $\alpha(t)$ which is differentiated in the interval $(0,1)$ and $0<\alpha(t)<1$

such that

$$
f\left(t, \lambda u, \frac{1}{\lambda} v\right) \geq \lambda^{\alpha(\lambda)} f(t, u, v), \forall t \in[0,1], \lambda \in(0,1), u, v \in[0,+\infty)
$$

Then the problem

$$
\left\{\begin{array}{l}
-D_{0+}^{\alpha} u(t)=f(t, u(t), u(t)), 0<t<1, \\
u(0)=u(1)=0
\end{array}\right.
$$

has a unique positive solution $u^{*}$ in $P_{w}$, where $w(t)=t^{\alpha-1}(1-t)$,

$t \in[0,1]$. Moreover, for any initial value $x_{0}, y_{0} \in P_{h}$, constructing successively the iterative scheme

$$
\begin{aligned}
& x_{n}(t)=\int_{0}^{1} G(t, s) f\left(s, x_{n-1}(s), y_{n-1}(s)\right) \mathrm{d} s, \quad n=1,2, \cdots, \\
& y_{n}(t)=\int_{0}^{1} G(t, s) f\left(s, y_{n-1}(s), x_{n-1}(s)\right) \mathrm{d} s, \quad n=1,2, \cdots,
\end{aligned}
$$

we have $x_{n} \rightarrow u^{*}$ and $y_{n} \rightarrow u^{*}$ as $n \rightarrow \infty$, where $G(t, s)$ is given as (10).

From Theorem 2.4, we can easily obtain the following result.
Theorem 3.5 Assume that (H1), (H2) hold and (H5) there exists the function $\alpha(t)$ which is differentiated in the interval $(0,1)$ and $0<\alpha(t)<1$ such that

$$
g(t, \mu u) \geq \mu^{\alpha(\mu)} g(t, u), \quad \mu \in(0,1), t \in[0,1], u \in[0,+\infty)
$$

and

$f\left(t, \lambda u, \frac{1}{\lambda} v\right) \geq \lambda f(t, u, v), \forall t \in[0,1], \lambda \in(0,1), u, v \in[0,+\infty)$

(H6) there exists a constant $\delta_{0}>0$ such that $f(t, u, v) \leq \delta_{0} g(t, u), t \in[0,1], u, v \geq 0$.

Then the problem (8) has a unique positive solution $u *$ in $P_{w}$, where $w(t)=t^{\alpha-1}(1-t), t \in[0,1]$. Moreover, for any initial value $x_{0}, y_{0} \in P_{w}$, constructing successively the iterative scheme

$$
\begin{aligned}
& x_{n}(t)=\int_{0}^{1} G(t, s) f\left(s, x_{n-1}(s), y_{n-1}(s)\right) \mathrm{d} s+\int_{0}^{1} G(t, s) g\left(s, x_{n-1}(s)\right) \mathrm{d} s, \quad n=1,2, \cdots, \\
& y_{n}(t)=\int_{0}^{1} G(t, s) f\left(s, y_{n-1}(s), x_{n-1}(s)\right) \mathrm{d} s+\int_{0}^{1} G(t, s) g\left(s, y_{n-1}(s)\right) \mathrm{d} s, \quad n=1,2, \cdots,
\end{aligned}
$$

we have $x_{n} \rightarrow u^{*}$ and $y_{n} \rightarrow u^{*}$ as $n \rightarrow \infty$, where $G(t, s)$ is given as (10).

\section{Acknowledgements}

This research was supported by A Project Supported by Scientific Research Fund of Sichuan Provincial Education Department (17ZB0370).

\section{References}

[1] D. J. Guo, V. Lakshmikantham, Coupled fixed points of nonlinear operators with applications, Nonlinear Analysis: Theory, Methods \& Applications, 11 (5), 1987, 623-632.

[2] D. J. Guo, V. Lakshmikantham, Nonlinear Problems in Abstract Gones ( Boston: Academic Press, 2014).
[3] D. J. Guo, Existence and uniqueness of positive fixed point for mixed monotone operators with applications, Applicable Analysis, 46 (1-2), 1992, 91-100.

[4] Z. T. Zhang, New fixed point theorems of mixed monotone operators and applications, Journal of Mathematical Analysis and Applications, 204 (1), 1996, 307-319.

[5] X. G. Lian, Y. J. Li, Fixed point theorems for a class of mixed monotone operators with Applications, Nonlinear Analysis: Theory, Methods \& Applications, 67 (9), 2007, 2752-2762.

[6] Z. Q. Zhao, Existence and uniqueness of fixed points for some mixed monotone operators, Nonlinear Analysis: Theory, Methods \& Applications, 73 (6), 2010, 1481-1490.

[7] C. B. Zhai, W. P. Yan, C. Yang, A sum operator method for the existence and uniqueness of positive solutions to RiemannLiouville fractional differential equation boundary value problems, Communications in Nonlinear Science and Numerical Simulation, 18 (4), 2013, 858-866. 
[8] J. Harjani, B. López, K. Sadarangani, Fixed point theorems for mixed monotone operators and applications to integral equations, Nonlinear Analysis: Theory, Methods \& Applications, 74 (5), 2011, 1749-1760.

[9] C. B. Zhai, L. L. Zhang, New fixed point theorems for mixed monotone operators and local existence-uniqueness of positive solutions for nonlinear boundary value problems, Journal of Mathematical Analysis and Applications, 382 (2), 2011, 594614.

[10] Z. D. Liang, L. L. Zhang, S. J. Li, Fixed point theorems for a class of mixed monotone operators, Zeitschrift für Analysis und ihre Anwendungen, 22 (3), 2003, 529-542.

[11] Y. Wu, Z. Liang, Existence and uniqueness of fixed points for mixed mono-tone operators with applications, Nonlinear Analysis: Theory, Methods \& Applications, 65 (10), 2006, 1913-1924.

[12] C. B. Zhai, M. R. Hao, Fixed point theorems for mixed monotone operators with perturbation and applications to fractional differential equation boundary value problems, Nonlinear Analysis: Theory, Methods \& Applications, 75 (4), 2012, 2542-2551

[13] M. A. Krasnosel' skii, Positive Solutions of Operators Equations (Noordoff : Groningen, 1964).

[14] Z. Zhao, X. Du, Fixed points of generalized e-concave (generalized e-convex) operators and their applications, Journal of Mathematical Analysis and Applications, 334 (2), 2007, 1426-1438.

[15] C. B. Zhai, C. M. Guo, On $\alpha$-convex operators, Journal of Mathematical Analysis and Applications, 316 (2), 2006, 556565.

[16] C. B. Zhai, C. Yang, C. M. Guo, Positive solutions of operator equation on ordered Banach spaces and applications, Computers \& Mathematics with Applications, 56 (12), 2008, $3150-3156$.

[17] Y. Wu, Z. Liang, Existence and uniqueness of fixed points for mixed monotone operators with applications, Nonlinear Analysis: Theory, Methods \& Applications, 65 (10), 2006,
$1913-1924$.

[18] I. Podlubny, Fractional Differential Equations, Mathematics in Science and Engineering (New York: Academic Press, 1999).

[19] S. G. Samko, A. A. Kilbas, O. I. Marichev, Fractional Integral and Derivatives ( Switzerland: Gordon and Breach, 1993).

[20] Z. B. Bai, Boundary value problem of fractional differential equation theory and Application (Beijing: China Science and Technology Press, 2013). (in Chinese).

[21] Z. Bai, H. Lü, Positive solutions for boundary value problem of nonlinear fractional differential equation, Journal of Mathematical Analysis and Applications, 311 (2), 2005, 495505.

[22] D. Jiang, C. Yuan, The positive properties of the Green function for Dirichlet-type boundary value problems of nonlinear fractional differential equations and its application, Nonlinear Analysis: Theory, Methods \& Applications, 72 (2), 2010, 710 719.

[23] X. Ding, Y. Feng, R. Bu, Existence, nonexistence and multiplicity of positive solutions for nonlinear fractional differential equations, Journal of Applied Mathematics and Computing, 40, 2012, 371-381.

[24] S. Liang, Positive Solutions for Singular Boundary Value Problem with Fractional q-Differences, Bulletin of the Malaysian Mathematical Sciences Society, 38 (2), 2015, 647666.

[25] K. Zhao, J. Liu, Multiple monotone positive solutions of integral BVPs for a higher-order fractional differential equation with monotone homomorphism, Advances in Difference Equations, 2016 (1), 2016, 1-17.

[26] L. Liu, X. Zhang, J. Jiang, Y. Wu, The unique solution of a class of sum mixed monotone operator equations and its application to fractional boundary value problems, Journal of Nonlinear Sciences and Application, 2016 (9), 2943-2958.

[27] D. Wardowski, Mixed monotone operators and their application to integral equations, Journal of Fixed Point Theory and Applications, 19 (2), 2017, 1103-1117. 